EPJ Web of Conferences 43, 05002 (2013)

DOI: $10.1051 /$ epjconf/20134305002

(C) Owned by the authors, published by EDP Sciences, 2013

\title{
White dwarf cooling sequences and cosmochronology
}

\author{
J. Isern ${ }^{1,2, a}$, A. Artigas ${ }^{3}$ and E. García-Berro 2,4 \\ ${ }^{1}$ Institut de Ciències de l'Espai (CSIC), Facultat de Ciències, Campus UAB, Torre C5-parell, \\ 08193 Bellaterra, Spain \\ ${ }^{2}$ Institute for Space Studies of Catalonia, c/Gran Capità 2-4, Edif. Nexus 104, \\ 08034 Barcelona, Spain \\ ${ }^{3}$ CRECIM, Universitat Autònoma de Barcelona, Campus UAB, 08193 Bellaterra, Spain \\ ${ }^{4}$ Departament de Física Aplicada, Universitat Politècnica de Catalunya, c/Esteve Terrades, 5, \\ 08860 Castelldefels, Spain
}

\begin{abstract}
The evolution of white dwarfs is a simple gravothermal process. This means that their luminosity function, i.e. the number of white dwarfs per unit bolometric magnitude and unit volume as a function of bolometric magnitude, is a monotonically increasing function that decreases abruptly as a consequence of the finite age of the Galaxy. The precision and the accuracy of the white dwarf luminosity functions obtained with the recent large surveys together with the improved quality of the theoretical models of evolution of white dwarfs allow to feed the hope that in a near future it will be possible to reconstruct the history of the different Galactic populations.
\end{abstract}

\section{INTRODUCTION}

White dwarfs represent the last evolutionary stage of low and intermediate mass stars, i.e. stars with masses smaller than $10 \pm 2 M_{\odot}$. Most of them are composed of carbon and oxygen, but white dwarfs with masses smaller than $0.4 M_{\odot}$ are made of helium and are members of close binary systems, while those more massive than $\sim 1.05 M_{\odot}$ are probably made of oxygen and neon. The exact composition of the cores of carbon-oxygen white dwarfs critically depends on the evolution during the previous red giant and asymptotic giant branch phase, and more specifically on the competition between the carbon$\alpha$ and triple- $\alpha$ reactions, on the details of the stellar evolutionary codes and on the choice of several other nuclear cross sections. In a typical case - for instance a white dwarf of $0.58 M_{\odot}$ — the total amount of oxygen represents the $62 \%$ of the total mass while its concentration in the central layers of the white dwarf can be as high as $85 \%$.

In all cases, the core is surrounded by a thin layer of pure helium with a mass ranging from $10^{-2}$ to $10^{-4} M_{\odot}$. This layer is, in turn, surrounded by an even thinner layer of hydrogen with a typical mass lying in the range of $10^{-4}$ to $10^{-15} M_{\odot}$. This layer is missing in $\sim 25 \%$ of the cases. From the phenomenological point of view, white dwarfs containing hydrogen are classified as DA while the remaining ones (the collectively denoted as non-DA) are classified as DO, DB, DQ, DZ and $\mathrm{DC}$, depending on their spectral features. The origin of these spectral differences and the relationship among them has not been yet elucidated, although it is related to the initial conditions during the AGB evolutionary phase, and also to delicate interplay between several physical process, among which we mention gravitational and thermal diffusion, radiative levitation, convection at the $\mathrm{H}-\mathrm{He}$ and $\mathrm{He}-$ core

\footnotetext{
a e-mail: isern@ieec.cat
}

This is an Open Access article distributed under the terms of the Creative Commons Attribution License 2.0, which permits unrestricted use, distribution, and reproduction in any medium, provided the original work is properly cited. 


\section{EPJ Web of Conferences}

interfaces, proton burning, stellar winds and mass accretion from the interstellar medium — see, for instance Fontaine (2013), this volume.

The structure of white dwarfs is sustained by the pressure of degenerate electrons and these stars cannot obtain energy from thermonuclear reactions. Therefore, their evolution can be described in terms of a simple cooling process [1] in which the internal degenerate core acts as a reservoir of energy and the outer non-degenerate layers control the energy outflow. A simple calculation indicates that the time they take to fade and disappear beyond the capabilities of the present telescopes is very long, $\sim 10$ Gyr. Thus the populations of white dwarfs retain important information about the past history of our Galaxy. In particular, they allow to obtain the age of the different Galactic components, namely the disk, the spheroid and the system of open and globular clusters, as well as the star formation history of the Galactic disk [2-7].

The tool to obtain such information is the luminosity function, which is defined as the number of white dwarfs of a given luminosity per unit volume and magnitude interval:

$$
N(l)=\int_{M_{\mathrm{i}}}^{M_{\mathrm{s}}} \Phi(M) \Psi\left[T-t_{\mathrm{cool}}(l, M)-t_{\mathrm{PS}}(M)\right] \tau_{\mathrm{cool}}(l, M) d M
$$

where $T$ is the age of the population under study, $l=-\log \left(L / L_{\odot}\right), M$ is the mass of the parent star (for convenience all white dwarfs are labeled with the mass of the main sequence progenitor), $t_{\text {cool }}$ is the cooling time down to luminosity $l, \tau_{\mathrm{cool}}=d t / d M_{\mathrm{bol}}$ is the characteristic cooling time, $M_{\mathrm{s}}$ is the maximum mass of a main sequence star able to produce a white dwarf, and $M_{\mathrm{i}}$ is the minimum mass of the main sequence stars able to produce a white dwarf of luminosity $l$, i.e. is the mass that satisfies the condition $T=t_{\text {cool }}(l, M)+t_{\mathrm{PS}}(M)$ and $t_{\mathrm{PS}}$ is the lifetime of the progenitor of the white dwarf. The remaining quantities, the initial mass function, $\Phi(M)$, and the star formation rate, $\Psi(t)$, are not known a priori and depend on the astronomical properties of the stellar population under study. Since the total density of white dwarfs of a given population is not usually well known, to compare the theoretical and observational luminosity functions it is customary to normalize the computed luminosity function to the bin with the smallest error bar. For instance, for the case of the disk white dwarf luminosity function it is traditionally chosen to normalize at $l=3$ [5]. In summary, if the observed luminosity function and the evolutionary behavior of white dwarfs are well known, it is possible to obtain the age and the star formation rate of the evolution under study.

\section{THE OBSERVED LUMINOSITY FUNCTIONS}

The first luminosity function was derived about four decades ago [13], and since then it has been noticeably improved with the work of many authors — see Fig. 2. The monotonic behavior of this function clearly proves that the evolution of white dwarfs is a simple gravothermal process, while the sharp cut-off at low luminosities is the consequence of the finite age of the Galaxy. The recent availability of data from the Sloan Digital Sky Survey (SDSS) has noticeably improved the accuracy of the new luminosity functions, as many new white dwarfs were added to the very limited sample of white dwarfs with measured magnitudes, parallaxes and proper motions. For instance, the white dwarf luminosity function of Ref. [14] (HA-LF) was built from a sample of $\sim 6,000$ DA and non-DA white dwarfs with accurate photometry and proper motions obtained from the SDSS Data Release 3 and the USNO-B catalogue, whereas that of Ref. [15] (DG-LF) was constructed from a sample of 3,528 spectroscopically-identified DA white dwarfs obtained from the SDSS Data Release 4.

The discrepancies between the HA-LF and the DG-LF at low luminosities are well understood, and can be attributed to the different way in which the effective temperatures and gravities of the sample of white dwarfs were observationally determined [15]. Furthermore, the DG-LF only considers DA white dwarfs and, at low temperatures, it is difficult to separate them from non-DA white dwarfs. 
Ageing Low Mass Stars: From Red Giants to White Dwarfs

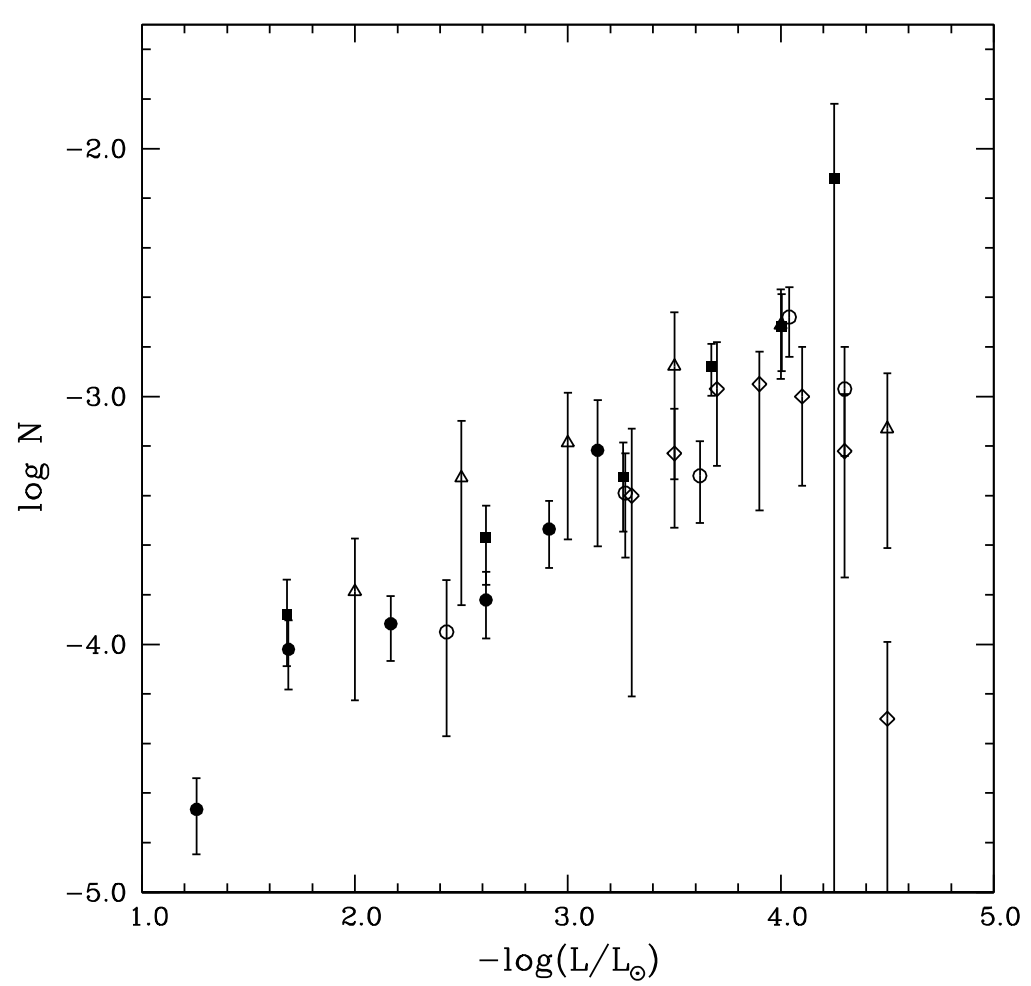

Figure 1. Luminosity functions obtained before the era of large surveys. The different symbols represent different determinations: full circles [8], full squares [9], open triangles [10], open diamonds [11], open circles [12].

For this reason in this work we will restrict ourselves to analyze the DG-LF for magnitudes smaller than $M_{\text {bol }} \sim 13$.

At high luminosities, say magnitudes smaller than $M_{\text {bol }} \sim 6$, the dispersion of both luminosity functions is very large - see Fig. 2. The reason is that both luminosity functions have been built using the reduced proper motion method that is not appropriate for bright white dwarfs. The UV-excess technique has allowed to build a luminosity function in the range -0.75 to 7 (KZ-LF) [16]. This method, however, is not adequate for dim stars and becomes rapidly incomplete out of this range of magnitudes. Fortunately, this sample overlaps with the HA-LF and, assuming continuity, it is possible to extend the luminosity function to the brightest region.

One of the potential problems of the luminosity functions obtained from the SDSS results from the fact that the integration time is fixed and, consequently, the $\mathrm{S} / \mathrm{N}$ ratio depends on the brightness of the source. This can lead to large uncertainties in the determination of the parameters of faint white dwarfs, and may introduce systematic errors [18]. Fortunately, a completely independent luminosity function has been obtained [17] from the SuperCOSMOS Sky Survey, which culls white dwarfs using their proper motion. As can be seen in Fig. 2, this luminosity function (hereafter called RH-LF) does not excessively deviate from the HA-LF at low luminosities, thus providing some hope that these luminosity functions are not affected by large systematic effects. However, for bright white dwarfs this luminosity function suffers from the same drawbacks as the HA-LF and the DG-LF luminosity functions. Thus, at bright luminosities is better to use the KZ-LF data.

Because of the overlap in the velocity distributions, the luminosity function obtained from reduced proper motion methods are in fact a superposition of thin and thick disc objects. It has been recently shown [17] that, in principle, it is possible to separate both populations using kinematic arguments. 


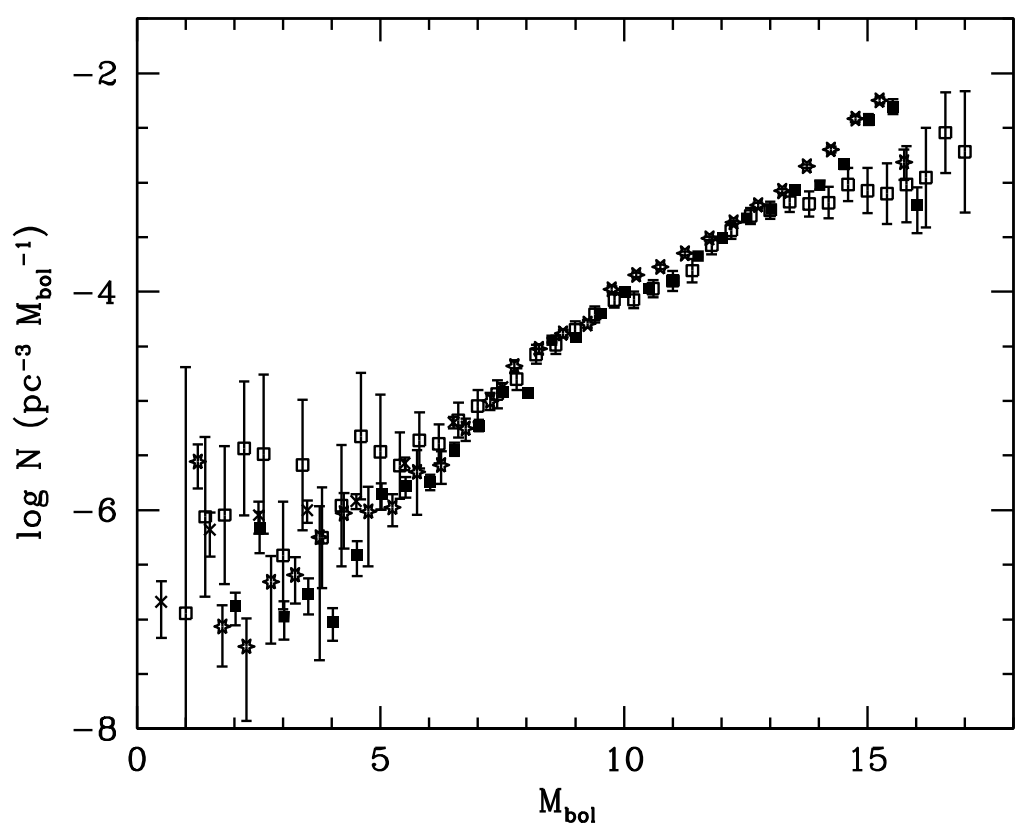

Figure 2. Luminosity functions obtained from large surveys. Solid squares: SDSS, all spectral types [14], hollow squares: SDSS, only DA white dwarfs [15], crosses: SDSS, hot DA white dwarfs [16], stars: SuperCOSMOS Sky Survey [17].

This technique yielded, for the first time, luminosity functions for both populations in a self-consistent way, thus offering the possibility to provide interesting insight in the sequence of events that led to the formation of the galactic disk — see below.

\section{AN OVERALL VIEW OF WHITE DWARF COOLING}

Since white dwarfs are degenerate objects, they cannot obtain energy from nuclear burning. Therefore, their evolution can be considered as a simple cooling process. Globally, the evolution of their luminosity can be written as:

$$
L+L_{v}=-\int_{0}^{M_{\mathrm{WD}}} C_{\mathrm{v}} \frac{d T}{d t} d m-\int_{0}^{M_{\mathrm{WD}}} T\left(\frac{\partial P}{\partial T}\right)_{V, X_{0}} \frac{d V}{d t} d m+\left(l_{\mathrm{s}}+e_{\mathrm{s}}\right) \dot{M}_{\mathrm{s}}
$$

where the 1.h.s. of the equation represents the sinks of energy, photons and neutrinos, while the r.h.s. contains the sources of energy, the heat capacity of the star, the compressional work, the contribution of the latent heat release and of the energy released by gravitationl settling upon crystallization, times the rate of crystallization, $\dot{M}_{\mathrm{s}}$ [5]. This equation has to be complemented with a relationship connecting the temperature of the core with the luminosity of the star.

The evolution of white dwarfs from the planetary nebula phase to its disappearance can be roughly divided in four stages:

Neutrino cooling: The range of luminosities of this phase is $\log \left(L / L_{\odot}\right)>-1.5$. This stage is very complicated because of the dependence on the initial conditions of the newly born star as well as on the complex and not yet well understood behavior of the hydrogen envelope. If the hydrogen layer is smaller than a critical value, $M_{\mathrm{H}} \leq 10^{-4} M_{\odot}$, nuclear burning via the pp-reactions quickly drops as the star cools down and never becomes dominant. Since astero-seismological observations seem to constrain 


\section{Ageing Low Mass Stars: From Red Giants to White Dwarfs}

the size of $M_{\mathrm{H}}$ well below this critical value, this source can be neglected. Fortunately, when neutrino emission becomes dominant, the different thermal structures converge to a unique one, granting the uniformity of the models with $\log \left(L / L_{\odot}\right) \leq-1.5$. Furthermore, since the time necessary to reach this value is $\leq 8 \times 10^{7}$ years for any model, its influence in the total cooling time is negligible [19], except of course at large luminosities.

Fluid cooling: This phase occurs at luminosities $-1.5 \geq \log \left(L / L_{\odot}\right) \geq-3$. The main source of energy is the gravothermal one. Since the plasma is not very strongly coupled $(\Gamma<179)$, its properties are reasonably well known. Furthermore, the flux of energy through the envelope is controlled by a thick non degenerate layer with an opacity dominated by hydrogen (if present) and helium, and weakly dependent on the metal content. The main source of uncertainty is related to the chemical structure of the interior, which depends on the adopted rate of the ${ }^{12} \mathrm{C}(\alpha, \gamma){ }^{16} \mathrm{O}$ reaction and on the treatment given to semiconvection and overshooting. If this rate is high, the oxygen abundance is higher in the center than in the outer layers, thus resulting in a reduced specific heat at the central layers of the star, where the oxygen abundance can be as high as $X_{\mathrm{O}}=0.85$ [20].

Crystallization: White dwarfs with $\log \left(L / L_{\odot}\right)<-3$ are expected to experience a first order phase transition, and their deep cores crystallize at these luminosities. Crystallization introduces two new sources of energy: latent heat and sedimentation. In the case of Coulomb plasmas, the latent heat is small, $\sim k_{\mathrm{B}} T_{\mathrm{s}}$ per nuclei, where $k_{\mathrm{B}}$ is the Boltzmann constant and $T_{\mathrm{s}}$ is the temperature of solidification. Its contribution to the total luminosity is small, $\sim 5 \%$, but not negligible [21]. During the crystallization process, the equilibrium chemical compositions of the solid and liquid plasmas are not equal. Therefore, if the resulting solid flakes are denser than the liquid mixture, they sink towards the central region. If they are lighter, they rise upwards and melt where the solidification temperature, which depends on the density, becomes equal to that of the isothermal core. The net effect is a migration of the heavier elements towards the central regions with the subsequent release of gravitational energy [22, 23]. Of course, the efficiency of the process depends on the detailed chemical composition and on the initial chemical profile and it is maximum for a mixture made of half oxygen and half carbon uniformly distributed through all the star [24]. An additional source of energy that has to be taken into account is the gravitational diffusion of ${ }^{22} \mathrm{Ne}$ synthesized from the initial content of ${ }^{12} \mathrm{C},{ }^{14} \mathrm{~N}$ and ${ }^{16} \mathrm{O}$ during the He-burning phase [25].

Debye cooling: When almost all the star has solidified, the specific heat follows Debye's law. However, the outer layers still have very large temperatures as compared with the Debye's one, and since their total heat capacity is still large enough, they prevent the sudden disappearance of the white dwarf at least for the case of white dwarfs with thick hydrogen envelopes [19].

\section{COOLING SEQUENCES}

In this work we have adopted the BASTI models ${ }^{1}$ [26]. These models can follow the diffusion of the different chemical species, convective mixing, residual nuclear burning and all the phenomena related with the crystallization process, and the evolutionary ages are in excellent agreement with other recent calculations [27]. The parameters for the envelopes adopted here are $q_{\mathrm{He}}=10^{-2} M_{\mathrm{WD}}$ and $q_{\mathrm{H}}=10^{-4} M_{\mathrm{WD}}$ for the DAs and $q_{\mathrm{He}}=10^{-3.5} M_{\mathrm{WD}}$ for the non-DAs.

The choice of the chemical composition of the white dwarf interior is of critical importance since all the factors influencing the cooling rate, specific heat, neutrino emission, crystallization temperature, sedimentation and so on, depend on the detailed chemical structure. In the present work, the chemical profile has been obtained assuming solar metallicity, convective overshooting during the main sequence and semiconvection during central He-burning, while the breathing pulses occurring at the end of the core He-burning have been suppressed [26]. The adopted rate for the ${ }^{16} \mathrm{C}(\alpha, \gamma){ }^{16} \mathrm{O}$ was that of Ref. [28].

\footnotetext{
1 These models can be downloaded from http://www. oa-teramo.inaf .it/BASTI.
} 


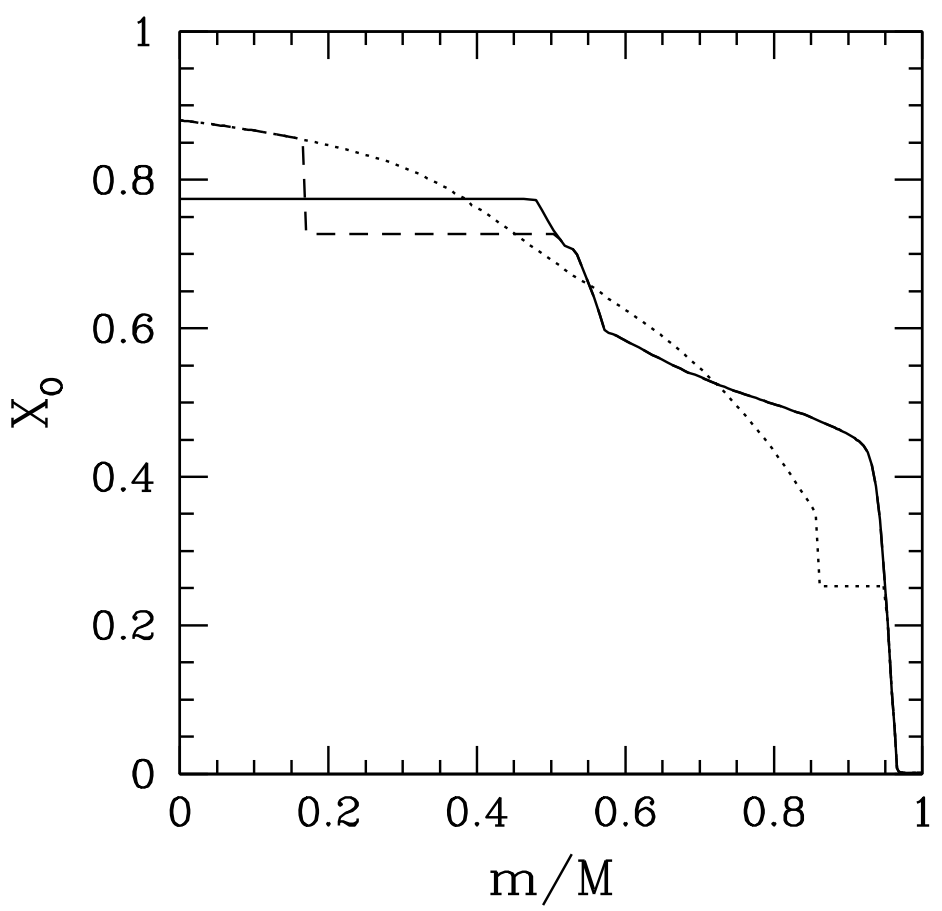

Figure 3. Oxygen profile of a $0.61 M_{\odot}$ white dwarf at the beginning of solidification (solid line) and when the $\sim 15 \%$ (dashed line) and $\sim 85 \%$ (dotted line) in mass has already crystallized.

Figure 3 displays the oxygen profiles for the $\mathrm{CO}$ core of a $\sim 0.6 M_{\odot}$ white dwarf progenitor obtained just at the end of the AGB phase (solid line). The inner part of the core, with a constant abundance of ${ }^{16} \mathrm{O}$, is determined by the maximum extension of the central He-burning convective region while beyond this region the oxygen profile is built when the thick He-burning shell is moving towards the surface. Simultaneously, gravitational contraction increases its temperature and density and, since the ratio between the ${ }^{16} \mathrm{C}(\alpha, \gamma){ }^{16} \mathrm{O}$ and the $3 \alpha$ reaction rates is smaller for higher temperatures, the oxygen mass fraction steadily decreases in the external part of the $\mathrm{CO}$ core [20]. Figure 3 also displays how the inner abundance of oxygen gradually increases in the inner regions as the crystallization front advances in mass. The region with a flat chemical profile placed just above the solidification front is due to the convective instability induced upon crystallization. The first calculation of a phase diagram for CO mixtures was done several years ago [29] and resulted in an eutectic shape. This result was a consequence of the assumption that the solid was entirely random. Later on [30], it was found that the $\mathrm{CO}$ phase diagram was of the spindle form. Because of this, the solid formed upon crystallization is richer in oxygen than the liquid and therefore denser. For a $0.6 M_{\odot}$ white dwarf with equal amounts of carbon and oxygen, $\delta \rho / \rho \simeq 10^{-4}$. Therefore the solid settles down at the core of the star and the lighter liquid left behind is redistributed by Rayleigh-Taylor instabilities [22, 23, 29, 31]. The result is an enrichment of oxygen in the central layers and its depletion in the outer ones. Even when rotation is considered, convection is indeed an efficient mechanism to redistribute the carbon rich fluid out from the crystallization front and that the liquid phase can be considered as well mixed [23].

Finally, we mention that the characteristic cooling time that appears in Eq. (1) not only depends on the internal energy sources or sinks but also on the photon luminosity which, in turn, depends on the transparency of the envelope. Since non-DA models are more transparent than the DA ones, they cool down much more rapidly as it can be seen in Fig. 4. 
Ageing Low Mass Stars: From Red Giants to White Dwarfs

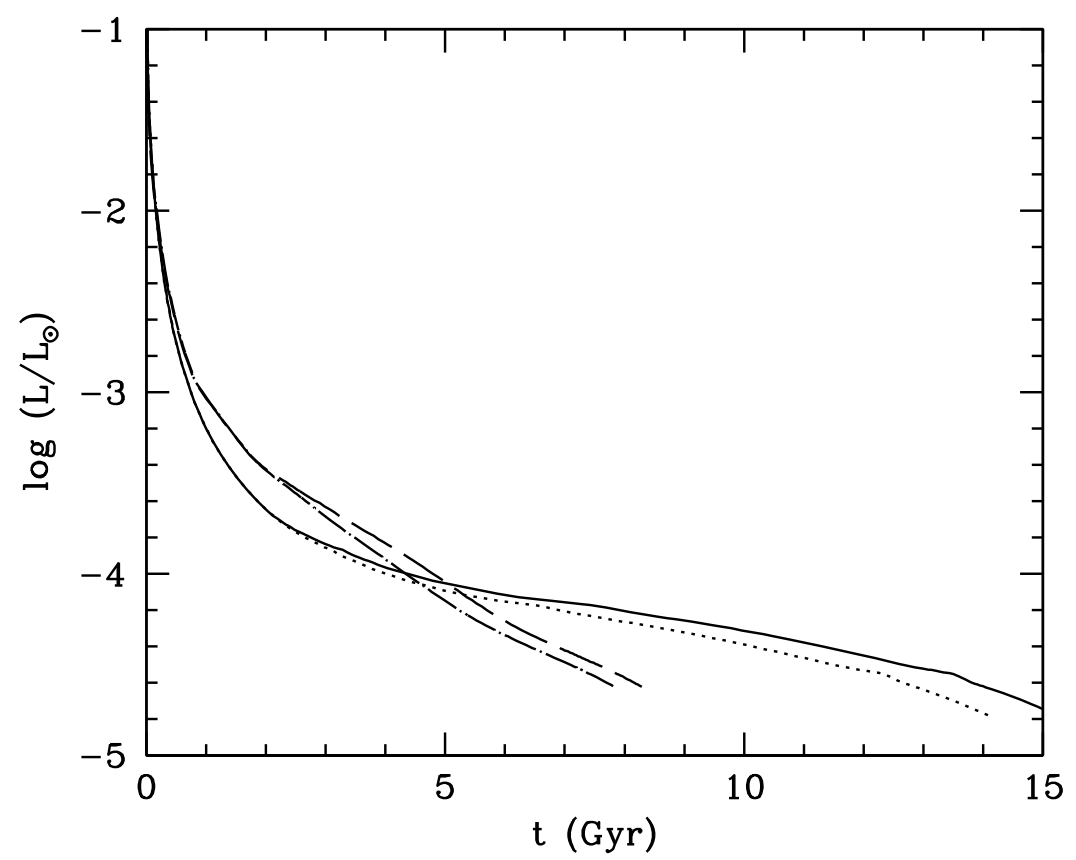

Figure 4. Time evolution of the luminosity of a $0.61 M_{\odot}$ white dwarf. The solid line corresponds to a DA model in which phase separation was included. The dotted line corresponds to the same model but disregarding phase separation. The dashed and the dotted-dashed lines represent, respectively, a non-DA model when separation is included and disregarded.

\section{THE AGE OF THE GALACTIC DISK}

A common picture of the formation of the Milky Way is that spiral galaxies form as a consequence of the gas cooling inside a spinning dark matter halo. In a first stage, the gas collapses in a dynamical time scale that lasts for several hundred million years, leaving behind a spherical stellar halo, and settles down into a disk from where stars form. Galactic discs are structures that are easily destroyed by mergers with other structures of similar mass. Therefore, if a disk appears almost undamaged, it means that its has not suffered strong mergers since it was born and that its life has been reasonably quiet. It is also possible for disks formed early in the life of a spiral galaxy to have been heated by minor mergers leading to the formation of a thick disk able to produce a new thin disk within it. According to this picture, the sequence of events leading to the formation of the presently observed structure of the Milky Way could have been the following one [32]: i) Formation of the primitive halo at $t \sim 12-13 \mathrm{Gyr}$, ii) Episodes of minor mergers of satellite systems at $t \sim 11-12 \mathrm{Gyr}$, iii) Formation of the disk at $t \sim 10-11 \mathrm{Gyr}$, iv) A major merger produces the formation of a thick disk at $t \sim 9-10 \mathrm{Gyr}$, and v) Formation of the thin disk at $t \sim 8$ Gyr.

In the case of the halo, the age is essentially determined by the age of the system of globular clusters, which at present is estimated to be $\sim 13 \mathrm{Gyr}$. In the case of the disk, several indicators are used. The main ones are the ages of $\mathrm{F}$ and $\mathrm{G}$ stars, the luminosity function of white dwarfs, radioactive clocks are also often employed, and finally the ages of old open clusters. Some of these ages are obtained from objects in the solar neighborhood, as it is the case of white dwarfs, assuming they are representative of the total Galaxy, which is not the case. On the contrary, old open clusters are distributed all over the Galaxy, and are considered more representative but, since their lifetime is relatively short (several $10^{8}$ years) many of them could have been destroyed and, in fact they only provide a lower limit to the 


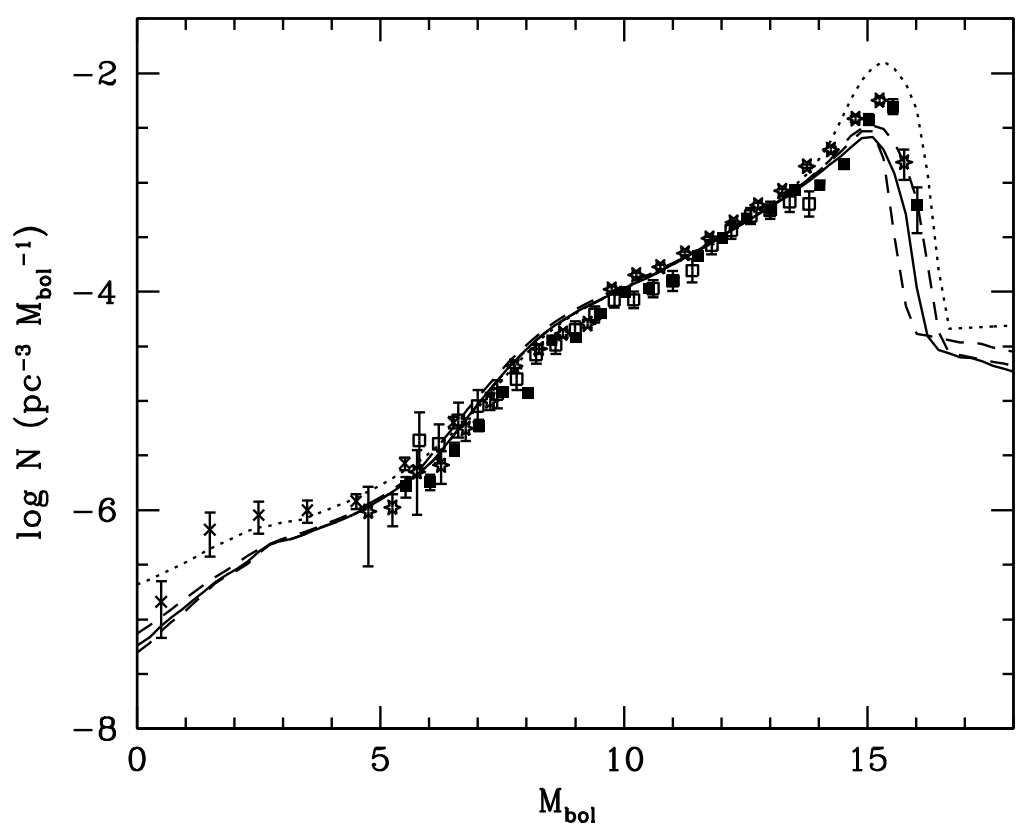

Figure 5. White dwarf luminosity function for different star formation rates and different ages of the Galactic disk. The symbols corresponding to the observational data are those of Fig. 2. Dashed lines, from left to right, constant SFR, and $t_{\text {disk }}=10,13$ Gyr. Solid line, $\Psi \propto\left(1+\exp \left[\left(t-t_{0}\right) / \tau\right]\right)^{-1}, \tau=3 \mathrm{Gyr}, t_{0}=10$ and $t_{\text {disk }}=13$ Gyr. Dotted line, $\Psi \propto \exp (-t / \tau), \tau=3 \mathrm{Gyr}, t_{\mathrm{disk}}=13 \mathrm{Gyr}$.

age of the disk, for which reason the local indicators continue to be extremely useful. Incidentally, it is worth noting that one of the key points in the determination of the age of the disk is NGC 6791, a very old, extremely metal-rich Galactic cluster. The age of this cluster estimated from the main-sequence turn-off method was different from the one estimated from the termination of the white dwarf cooling sequence. When the energy release due to the gravitational diffusion of ${ }^{22} \mathrm{Ne}$ and the settling of oxygen upon crystallization are included, both ages coincide and turn to be 8 Gyr [33].

There are two important properties of the luminosity function that deserve a comment. The first one is that, after normalization, the bright part of the luminosity function $\left(M_{\mathrm{bol}} \leq 14\right)$ is almost independent of the star formation rate [34], $N(l) \propto\left\langle\tau_{\text {cool }}\right\rangle$, unless a burst of star formation occurred very recently. The second important fact that needs to be considered is that Eq. (1) do not satisfies Piccard's theorem for the inversion of integral equations. Thus, the star formation rate cannot be directly obtained, as the unicity of the solution cannot be guaranteed and the final result depends on the trial function used to fit observations. The first property is clearly illustrated in Fig. 5, where the luminosity functions obtained using different SFRs are almost coincident in the region $6 \leq M_{\text {bol }} \leq 14$. The corollary is that the BASTI models reproduce reasonably well the evolution of white dwarfs in this region.

The age of the disk depends on the form adopted for the star formation rate. If a constant rate is adopted, the cutoff is compatible with an age of $\sim 13 \mathrm{Gyr}$, i.e. the disk would had been formed a short time after the primitive halo. If an exponentially declining star formation rate is adopted, it is necessary to reduce the age of the disk to $\sim 11 \mathrm{Gyr}$ (not shown in the figure) to adjust the position of the cut-off. A good fit can also be obtained if an almost constant rate lasting for the last $\sim 10$ Gyr preceded by an exponentially growing star formation activity is adopted. This is the same to say that the disk started to form from the center to the periphery. Notice that the cool end of the luminosity function still shows an important dispersion in the values and to elucidate among these possibilities it will be necessary to improve its accuracy at low luminosities. 


\section{Ageing Low Mass Stars: From Red Giants to White Dwarfs}

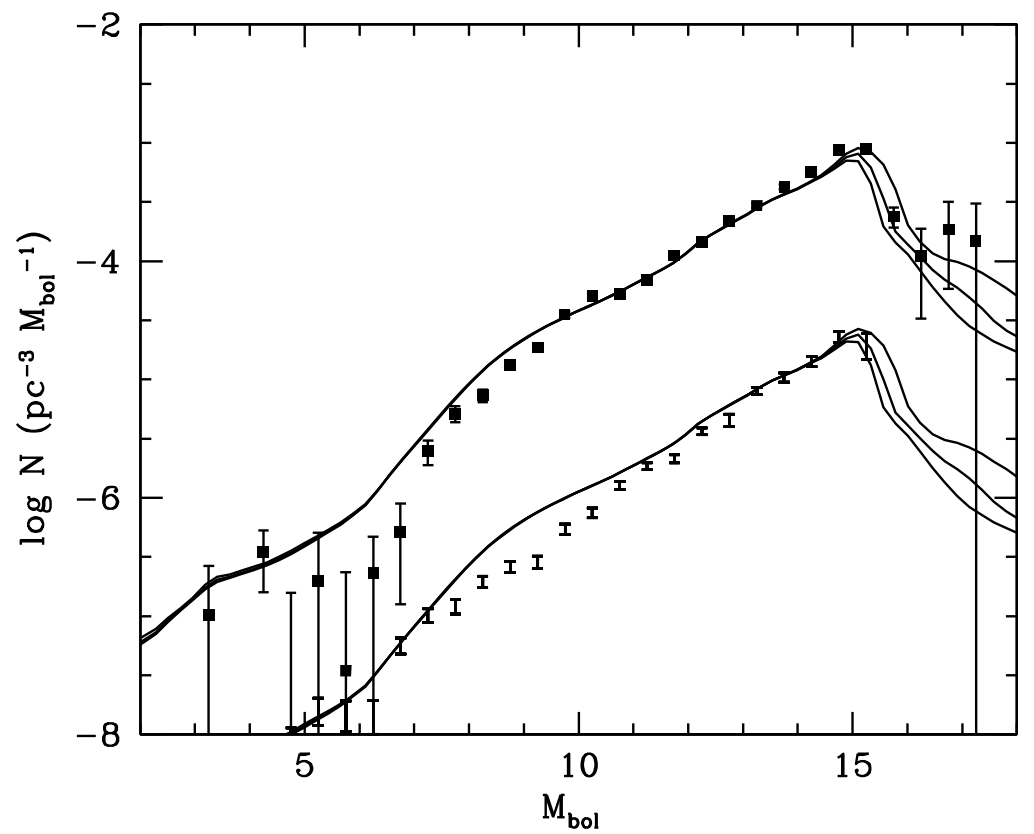

Figure 6. White dwarf luminosity function of the thin (upper curves) and thick (lower curves) disks [17]. The data corresponding to the thick disk have been shifted by a quantity of -1 for a sake of clarity. The theoretical function have been computed for a constant star formation rate and ages of 10,11 and $13 \mathrm{Gyr}$ (from left to right respectively).

Obviously, the determination of the ages of the thin and thick disks would be extremely helpful to prove the previously described sequence of events. Figure 6 displays the observed luminosity functions of the thin and thick disks, as well as the theoretical predictions assuming a constant star formation rate and different ages. The most striking feature is that both structures look as if they were coeval since the maximum of both distributions lies approximately at the same magnitude, $M_{\mathrm{bol}} \sim 15$. Furthermore, both populations seem to be rather old, $\sim 11 \mathrm{Gyr}$, in agreement with the luminosity functions obtained using the SDSS. Certainly, it is premature to extract conclusions since the cut-off of the thick disk is not well defined and the modeling of very old white dwarfs is still plenty of uncertainties. Nevertheless, if this result turns out to be correct, it could be obviously interpreted as if there was no difference between the time at which thin and the thick disk formed, and moreover that this unique disk formed quite soon in life of the Milky Way. This, if correct, could give support to the recent discovery that sub-populations with similar $[\alpha / \mathrm{Fe}]-[\mathrm{Fe} / \mathrm{H}]$ have a smooth distribution of scale heights, thus suggesting that effectively there is not a distinctive thick disk population. It is also important to notice here that in deriving the luminosity function it has been assumed that no vertical and radial migration of stars is effective. If these effects were important, it would be necessary to compute the luminosity function in the context of a complete numerical simulation of the Galaxy.

\section{CONCLUSIONS}

The use of white dwarfs as cosmochronometers has experienced noticeable advances during the last years both from the theoretical and observational point of views, and has become a reliable tool to measure the age of an ensemble population of stars if the conditions are well defined, as is the case, for instance, of NGC 6791. Furthermore, having for the first time separated luminosity functions of the thin and thick disk opens new possibilities to understand the origin and evolution of the Milky Way. 
However, several unsolved problems still remain. From the theoretical point of view, there are still noticeable differences among the different cooling tracks at low luminosities. These differences are probably due to the use of different boundary conditions [35] and different sizes and physics adopted for the envelope. An additional problem is our incomplete understanding of the origin and evolution of the DA, non-DA character, which could introduce some uncertainties in the determinations of the theoretical luminosity function. From the observational point of view the main problem resides in the still poor determination of the luminosity function of cool white dwarfs, as well as in the criteria to efficiently

separate the different populations (thin disk, thick disk and spheroid) of Galactic white dwarfs.

This research was partially supported by MICINN grants AYA2011-24704, and AYA2011-23102 by the ESF EUROGENESIS project (MICINN grants EUI2009-04167 and 04170), by the European Union FEDER funds and by the AGAUR.

\section{References}

[1] L. Mestel, MNRAS 112, 583 (1952)

[2] L.G. Althaus, A.H. Córsico, J. Isern, E. García-Berro, AAPR 18, 471 (2010)

[3] G. Fontaine, P. Brassard, P. Bergeron, PASP 113, 409 (2001)

[4] B.M.S. Hansen, J. Liebert, ARAA 41, 465 (2003)

[5] J. Isern, E. García-Berro, M. Hernanz, R. Mochkovitch, Journal of Physics Condensed Matter 10, $11263(1998)$

[6] D. Koester, AAPR 11, 33 (2002)

[7] D. Koester, G. Chanmugam, Reports on Progress in Physics 53, 837 (1990)

[8] J. Liebert, C.C. Dahn, D.G. Monet, ApJ 332, 891 (1988)

[9] D.W. Evans, MNRAS 255, 521 (1992)

[10] T.D. Oswalt, J.A. Smith, M.A. Wood, P. Hintzen, Nature 382, 692 (1996)

[11] S.K. Leggett, M.T. Ruiz, P. Bergeron, ApJ 497, 294 (1998)

[12] R.A. Knox, M.R.S. Hawkins, N.C. Hambly, MNRAS 306, 736 (1999)

[13] V. Weidemann, ARAA 6, 351 (1968)

[14] H. Harris, AJ 131, 571 (2006)

[15] S. De Gennaro, T. von Hippel, D.E. Winget, S.O. Kepler, A. Nitta, D. Koester, L. Althaus, AJ 135, 1 (2008)

[16] J. Krzesinski, S.J. Kleinman, A. Nitta, S. Hügelmeyer, S. Dreizler, J. Liebert, H. Harris, A\&A 508, 339 (2009)

[17] N. Rowell, N.C. Hambly, MNRAS 417, 93 (2011)

[18] M.M. Limoges, P. Bergeron, ApJ 714, 1037 (2010)

[19] F. D’ Antona, I. Mazzitelli, ApJ 347, 934 (1989)

[20] M. Salaris, I. Dominguez, E. García-Berro, M. Hernanz, J. Isern, R. Mochkovitch, ApJ 486, 413 (1997)

[21] G. Shaviv, A. Kovetz, A\&A 51, 383 (1976)

[22] R. Mochkovitch, A\&A 122, 212 (1983)

[23] J. Isern, R. Mochkovitch, E. García-Berro, M. Hernanz, ApJ 485, 308 (1997)

[24] J. Isern, E. García-Berro, M. Hernanz, G. Chabrier, ApJ 528, 397 (2000)

[25] E. García-Berro, L.G. Althaus, A.H. Córsico, J. Isern, ApJ 677, 473 (2008)

[26] M. Salaris, S. Cassisi, A. Pietrinferni, P.M. Kowalski, J. Isern, ApJ 716, 1241 (2010)

[27] I. Renedo, L.G. Althaus, M.M. Miller Bertolami, A.D. Romero, A.H. Córsico, R.D. Rohrmann, E. García-Berro, ApJ 717, 183 (2010) 
[28] R. Kunz, M. Fey, M. Jaeger, A. Mayer, J.W. Hammer, G. Staudt, S. Harissopulos, T. Paradellis, ApJ 567, 643 (2002)

[29] D.J. Stevenson, Journal de Physique 41, C61 (1980)

[30] L. Segretain, G. Chabrier, M. Hernanz, E. García-Berro, J. Isern, R. Mochkovitch, ApJ 434, 641 (1994)

[31] L.G. Althaus, E. García-Berro, J. Isern, A.H. Córsico, M.M. Miller Bertolami, A\&A 537, A33 (2012)

[32] I.N. Reid, ARAA 43, 247 (2005)

[33] E. García-Berro, S. Torres, L.G. Althaus, I. Renedo, P. Lorén-Aguilar, A.H. Córsico, R.D. Rohrmann, M. Salaris, J. Isern, Nature 465, 194 (2010)

[34] J. Isern, S. Catalán, E. García-Berro, S. Torres, Journal of Physics Conference Series 172, 012005 (2009)

[35] R.D. Rohrmann, L.G. Althaus, E. García-Berro, A.H. Córsico, M.M. Miller Bertolami, A\&A 546, A119 (2012) 